\title{
ON AN OPTIMAL SHAPE DESIGN PROBLEM IN CONDUCTION
}

\author{
José Carlos Bellido ${ }^{1}$
}

\begin{abstract}
In this paper we analyze a typical shape optimization problem in two-dimensional conductivity. We study relaxation for this problem itself. We also analyze the question of the approximation of this problem by the two-phase optimal design problems obtained when we fill out the holes that we want to design in the original problem by a very poor conductor, that we make to converge to zero.
\end{abstract}

Mathematics Subject Classification. 49J45, 49Q10.

Received May 13, 2005.

\section{INTRODUCTION}

Consider an open, regular, simply-connected domain $\Omega \subset \mathbf{R}^{2}$, another open, regular domain $\omega \subset \Omega$ with $\partial \Omega \cap \partial \omega=\emptyset$ and $u_{0} \in H^{1}(\Omega)$. Let $u \in H^{1}(\Omega \backslash \omega)$ be the solution of the elliptic problem

$$
\begin{cases}-\operatorname{div}(\beta \nabla u)=0 & \text { in } \Omega \backslash \omega, \\ u=u_{0} & \text { on } \partial \Omega, \\ \beta \nabla u \cdot n=0 & \text { on } \partial \omega .\end{cases}
$$

We would like to analyze the shape optimization problem associated to the previous elliptic system, namely

$$
\text { Minimize } I(\omega)=\int_{\Omega \backslash \omega} \varphi(x, u(x), \nabla u(x)) \mathrm{d} x,
$$

over the $\omega$ 's verifying the previous conditions and subject to the volume constraint

$$
\int_{\omega} \mathrm{d} x=\delta
$$

where $0<\delta<|\Omega|$ is a data of the problem. A physical meaning of the problem can be to determine how to distribute a fixed amount of a given conducting material with conductivity $\beta$ in the domain $\Omega$ in order to minimize the given criterion $I$.

Keywords and phrases. Optimal shape design, relaxation, variational approach, $\Gamma$-convergence, semiconvex envelopes, quasiconvexity.

1 Mathematical Institute, University of Oxford, 24-29 St. Giles', OX1 3LB, Oxford, UK; JoseCarlos.Bellido@uclm.es (On leave from Universidad de Castilla-La Mancha (Spain).) 
We will assume the following typical bounds on $\varphi$

$$
c \leq \varphi(x, u, \lambda) \leq h_{1}(x, u)+h_{2}(x, u)|\lambda|^{2},
$$

where $c$ is a constant and $h_{1}, h_{2}$ are locally bounded functions with $h_{2} \geq 0$. It is not necessary to impose coercivity on $\varphi$ as it will be obtained from the state equation.

If we try to apply the direct method of the Calculus of Variations to the previous problem, we proceed taking a minimizing sequence of domains $\left\{\omega_{j}\right\}$, for this sequence we have the associated sequence of states $\left\{u_{j}\right\}$, and we need to extract a weakly convergent subsequence of $\left\{u_{j}\right\}$ in $H^{1}(\Omega)$ ( $\Omega$ is the reference domain). Each $u_{j}$ is the solution of an elliptic PDE at the domain $\Omega \backslash \omega_{j}$, and as $\omega_{j}$ is a Lipschitz domain, $u_{j}$ can be extended to a function in the space $H^{1}(\Omega)$, that we still denote the same. By the lower bound assumed on $\varphi$ we know that

$$
\left\|u_{j}\right\|_{H^{1}\left(\Omega \backslash \omega_{j}\right)}
$$

is uniformly bounded with respect to $j$, however it is not true in general that

$$
\left\|u_{j}\right\|_{H^{1}(\Omega)}
$$

is uniformly bounded uniformly with respect to $j$, as the norm of the extension operators for domains $\omega_{j}$ can blow up (and a hypothesis assuming this not to happen does not fit with typical microstructural configurations like laminates). Therefore, the first difficulty that we find in the analysis of this problem is that it is not coercive in the sense explained before. In the numerical practise to overtake this problem is very common to fill out the set of holes $\omega$ by a very poor conducting material with conductivity $\alpha>0$, with $\alpha$ small, so that the shape optimization problem is approximated by the two-phase optimal design problem

$$
\text { Minimize } I_{\alpha}(\omega)=\int_{\Omega}(1-\chi(x)) \varphi\left(x, u_{\alpha}(x), \nabla u_{\alpha}(x)\right) \mathrm{d} x
$$

subject to

$$
\int_{\Omega} \chi(x) \mathrm{d} x=\delta
$$

where $\chi$ is the characteristic function of $\omega$ and $u_{\alpha} \in H^{1}(\Omega)$ is the unique solution of

$$
\begin{cases}-\operatorname{div}\left((\alpha \chi(x)+(1-\chi(x)) \beta) \nabla u_{\alpha}\right)=0 & \text { in } \Omega, \\ u=u_{0} & \text { on } \partial \Omega .\end{cases}
$$

It is easy to prove that problem $\left(P_{\alpha}\right)$ is coercive but it still lacks optimal solutions because minimizing sequences of shapes become more and more intricate extending along the whole $\Omega$ and microstructure develops (see the pioneering work [20]). This yields the need of studying relaxation of the above problem. The main tool, very successful both theoretically and practically, to analyze relaxation for two-phase optimal design problems has been Homogenization Theory, this is known as the homogenization method in optimal design $($ see $[1,11,15,25])$. For instance, the homogenization method applies for obtaining a relaxation of the problem of mixing two conducting materials in order to minimize an integral functional, when the integrand does not depend on the gradients of the state, or it does in a suitable way (see $[24,25])$.

Coming back to the shape optimization problem, as we mentioned before it is rather frequent, and physically reasonable, to approximate problem $(P)$ by problem $\left(P_{\alpha}\right)$ in order to simulate optimal solutions, but there is not rigorous mathematical justification of this procedure in general. Hence an open, outstanding problem is to obtain the above optimal shape design problem as the degeneracy limit of the problem of mixing two materials as one of the materials degenerates to void. That is a very interesting $\Gamma$-convergence problem and, to the best of the author knowledge, very little seems to be known (see [1] where this approximation problem is proposed). There are however partial results in the elasticity and conductivity frameworks for the case of the 
compliance cost functional [1-4]. The fact that this functional is self-adjoint allows a suitable reformulation as an optimization problem in term of stresses and a convergence result is shown in this formulation.

Very recently a new approach to analyze relaxation for optimal design problems has been proposed. The approach is based on the introduction of a new variable in the form of a potential or stream function in order to avoid the non-local character of the state equation $\left(e_{\alpha}\right)$. Then we can reformulate the optimal design problem as a vector variational problem without differential constraints and study relaxation for this problem by usual techniques: computing the quasiconvexification of the integrand of the variational problem and studying relaxation in terms of Young measures. Using this idea fully explicit relaxations have been obtained for twomaterial optimal design problems, with cost functionals depending also on the gradient of the state, in twodimensions $[8,23]$, and in three-dimensions [6]. The idea of reformulating an elliptic PDE as a differential inclusion has been very successful in some other contexts, as for instance to provide counterexamples to the regularity of solutions of elliptic systems $[19,26]$, or to establish existence results for optimal design and optimal control problems $[7,9,10]$. In this paper we propose to apply this approach to the problem at hand. We carry out this program in the following steps:

1. firstly we study relaxation for the variational reformulation of problem $\left(P_{\alpha}\right)$, obtaining a fully explicit relaxation (we explicitly compute the semiconvex envelope of the involved density);

2. we analyze relaxation for the variational reformulation of problem $(P)$ with the purpose of understanding non-existence of optimal solutions for this problem. We again compute explicitly the semiconvex envelope of the energy density involved, and prove a partial result in the direction of a relaxation result. Indeed, what we prove is that the infima of the problem in terms of gradient Young measures, the problem in which we substitute the density $W$ by the computed semiconvex envelope and the original infimum, coincide. We do not prove that this two new problems admit minimizers, again we find the difficulty of the lack of coercivity;

3. we study the approximation of the 'relaxed' formulation of the shape optimization problem that we get, by the relaxed formulation obtained for the sequence of optimal design problems, as $\alpha \rightarrow 0$.

The reader should notice that the situation here is highly non-standard regarding lack of coercivity for this problem, and as a consequence, a lot of questions have still to be understood. However, we think that our approach here is original and that the results that we present are interesting in themselves and probably useful for future work on the subject.

The plan of the paper is as follows. In Section 2 we introduce the variational formulation of the problems $\left(P_{\alpha}\right)$ and $(P)$. In Section 3 we obtain a fully explicit relaxation of problem $\left(P_{\alpha}\right)$ by computing the constrained semiconvex envelope of the density involved in the variational reformulation. In Section 3 is also given the right definition of that semiconvex envelope. In Section 4 we analyze relaxation for problem $(P)$, and finally Section 5 is devoted to the analysis of the approximation problem.

\section{Variational Reformulations of problems $\left(P_{\alpha}\right)$ and $(P)$}

This section is devoted to the reformulation of problems $\left(P_{\alpha}\right)$ and $(P)$ as vector variational problems.

Let us begin by the reformulation of problem $\left(P_{\alpha}\right)$. If $u$ is the solution of equation $\left(e_{\alpha}\right)$ then there exists an stream function $v \in H^{1}(\Omega)$ such that

$$
(\alpha \chi(x)+\beta(1-\chi(x))) \nabla u(x)+T \nabla v(x)=0, \quad \text { a.e. } x \in \Omega,
$$

where $T$ is the counter-clockwise $\frac{\pi}{2}$-rotation [18]. If we call $\Lambda_{\gamma}$ (for any $\gamma \in \mathbf{R}$ ) to the manifold

$$
\Lambda_{\gamma}=\left\{B \in \mathbf{M}^{2 \times 2}: \gamma A^{(1)}+T A^{(2)}=0\right\},
$$

the identity (2) can be rewritten as the first order differential inclusion

$$
(\nabla u(x), \nabla v(x)) \in \Lambda_{\alpha} \cup \Lambda_{\beta},
$$


a.e. $x \in \Omega$, and this is equivalent to the $\operatorname{PDE}\left(e_{\alpha}\right)$. Now we can pick the two variables $u, v$ up in a single vector $U=(u, v)$ and define the densities

$$
\begin{gathered}
W_{\alpha}: \Omega \times \mathbf{R} \times \mathbf{M}^{2 \times 2} \rightarrow \mathbf{R}^{*}=\mathbf{R} \cup\{+\infty\}, \\
W_{\alpha}(x, U, A)= \begin{cases}\varphi\left(x, U^{(1)}, A^{(1)}\right) & \text { if } \beta A^{(1)}+T A^{(2)}=0, \\
0 & \text { if } \alpha A^{(1)}+T A^{(2)}=0, \\
+\infty & \text { otherwise, }\end{cases}
\end{gathered}
$$

and

$$
\begin{aligned}
& V_{\alpha}: \mathbf{M}^{2 \times 2} \rightarrow \mathbf{R}^{*}, \\
& V_{\alpha}(A)= \begin{cases}0 & \text { if } \beta A^{(1)}+T A^{(2)}=0 \text { and } \alpha A^{(1)}+T A^{(2)} \neq 0, \\
1 & \text { if } \alpha A^{(1)}+T A^{(2)}=0, \\
+\infty & \text { otherwise }\end{cases}
\end{aligned}
$$

where $U^{(i)}$ and $A^{(i)}$ are the $i$-th component and $i$-th row of $U$ and $A$ respectively, $i=1,2$.

In order to avoid contradiction in the definition of $W$ we assume that

$$
\varphi(x, u, 0)=0,
$$

a.e. $x \in \Omega$ and all $u \in \mathbf{R}$. In this case $W(x, u, \cdot)$ is continuous where is finite, however $W$ is not a Carathéodory function because it takes on the value $+\infty$ suddenly.

If we now consider the variational problem

$$
\text { Minimize } I(U)=\int_{\Omega} W_{\alpha}(x, U(x), \nabla U(x)) \mathrm{d} x,
$$

over the set

subject to the integral constraint

$$
\mathcal{U}=\left\{U \in H^{1}\left(\Omega ; \mathbf{R}^{2}\right): U^{(1)}=u_{0} \text { on } \partial \Omega\right\}
$$

$$
\int_{\Omega} V_{\alpha}(\nabla U(x)) \mathrm{d} x=\delta
$$

is not difficult to realize that problem $\left(P_{\alpha}\right)$ and $\left(P V_{\alpha}\right)$ are equivalent (see [10] for a rigorous proof in a general situation). With this formulation we avoid the state equation for the optimization problem $\left(P_{\alpha}\right)$, paying the price that we now have a vector variational problem.

The shape optimization problem $(P)$ can also be reformulated as a vector variational problem simply observing that the solution of the boundary value problem $(e)$ is also a solution (in the sense of distributions) of the degenerate elliptic equation

$$
\begin{cases}-\operatorname{div}\left(\chi_{\Omega \backslash \omega} \beta \nabla u\right)=0 & \text { in } \Omega \\ u=u_{0} & \text { on } \partial \Omega,\end{cases}
$$

and conversely. What we mean is that if $u$ is a solution of $(e)$ then $u$ extended to the hole $\Omega$ (this can be done because we are assuming $\omega$ Lipschitz) is a solution of $\left(e^{\prime}\right)$ and conversely if $u$ is a solution of $\left(e^{\prime}\right)$ (for this problem we do not have uniqueness) then $u$ restricted to $\Omega \backslash \omega$ is the solution of (e). Since the function $\chi_{\Omega \backslash \omega} \beta \nabla u$ is divergence-free we can proceed as before. In this case we reformulate problem $(P)$ as the vector variational problem

over the set

$$
\text { Minimize } I(U)=\int_{\Omega} W(x, U(x), \nabla U(x)) \mathrm{d} x,
$$

$$
\mathcal{U}=\left\{U \in H^{1}\left(\Omega ; \mathbf{R}^{2}\right): U^{(1)}=u_{0} \text { on } \partial \Omega\right\}
$$


subject to the integral constraint

$$
\int_{\Omega} V(\nabla U(x)) \mathrm{d} x=\delta
$$

where the densities $W$ and $V$ are defined in a analogue way to $W_{\alpha}$ and $V_{\alpha}$ simply changing the manifold $\Lambda_{\alpha}$ by $\Lambda_{0}=\left\{A^{(2)}=0\right\}$.

One can hope that variational problem $(P V)$ is equivalent to our optimal shape design problem $(P)$, however it is not, unless we impose the additional restrictions on the admissible functions of $\mathcal{U}$ that the sets

$$
\left\{x \in \Omega: \nabla U^{(2)}(x)=0\right\}
$$

are open and Lipschitz. This is a quite artificial and unreasonable hypothesis, so instead of assuming such a hypothesis we will analyze relaxation for problem $(P V)$. This means that we are not imposing any condition on the interface between the region with and without material, or in other words, we are dropping the restrictions on the boundary of the admissible shapes for the design problem, thus we are enlarging the set of admissible designs.

A word should be said about quasiconvexification here. In this paper we deal with functionals whose integrands are not Carathéodory functions and hence we will define the proper semiconvex envelope for relaxation of those variational problems as an infimum over a set of Young measures, instead of as an infimum over a set of gradients, which may be too rigid in this situation. Of course, as our integrands are not Carathédory functions, we cannot be sure that the infima in terms of Young measures or gradients coincide, but anyhow with the envelope defined in terms of Young measures we still have a relaxation result and this is our aim here. For the sake of rigorousness and to avoid confusion we will not use the word quasiconvexification here and we will refer to our envelopes as semiconvex envelopes.

\section{Relaxation of problem $\left(P V_{\alpha}\right)$}

As we have mentioned in the Introduction our approach for relaxation of the optimal design problem $\left(P_{\alpha}\right)$ consists in the relaxation of the equivalent formulation $\left(P V_{\alpha}\right)$. There are two delicate facts concerning relaxation for that special problem with respect to typical problems in the Calculus of Variations. The first one is that the integrand $W_{\alpha}$ is not a Carathéodory function as we have mentioned above, and the second one is the volume constraint required by the variational problem $\left(P V_{\alpha}\right)$. The first difficulty can be overtaken defining the appropriate semiconvex envelope in terms of Young measures associated to sequences of admissible gradients for the problem. This definition also allows to impose the volume constraint from the beginning in the semiconvex envelope, and therefore we can also tackle with the second difficulty. In this case we should talk then of constrained semiconvex envelope. The appropriate definition in this case would be

$$
W_{\alpha}^{\sharp}(x, U, A, t)=\inf \left\{\int_{\mathbf{M}^{2 \times 2}} W_{\alpha}(x, U, F) \mathrm{d} \nu(F): \nu \in \mathcal{A}_{\alpha}(A, t)\right\},
$$

where $\mathcal{A}_{\alpha}(A, t)$ is the set of homogenous gradient Young measures (see [21]) $\nu$ such that

$$
A=\int_{\mathbf{M}^{2 \times 2}} F \mathrm{~d} \nu(F)
$$

and the volume constraint

$$
t=\int_{\mathbf{M}^{2 \times 2}} V_{\alpha}(F) \mathrm{d} \nu(F) .
$$

This constrained "quasiconvexification" was introduced in [17,22], and for an optimal design problem of the kind of $\left(P_{\alpha}\right)$ but with a different cost functional (actually the cost functional was the square of the gradient of the state) was explicitly computed in [23] for the volume constrained case, and in [8] for the case without volume constraint. 
Before to state and prove the main result in this section we need to introduce a little bit of notation. Put

$$
\begin{aligned}
g_{\alpha}(B)= & (\alpha+\beta)^{2}\left[\left(\alpha B^{(1)}+T B^{(2)}\right) \cdot\left(\beta B^{(1)}+T B^{(2)}\right)\right]^{2} \\
& -4 \alpha \beta\left|\alpha B^{(1)}+T B^{(2)}\right|^{2}\left|\beta B^{(1)}+T B^{(2)}\right|^{2}, \\
h_{\alpha}(B)= & \left(\alpha B^{(1)}+T B^{(2)}\right) \cdot\left(\beta B^{(1)}+T B^{(2)}\right) .
\end{aligned}
$$

Let $r_{i}(B), i=1,2$, be

$$
\begin{aligned}
& r_{1}^{\alpha}(B)=\frac{1}{2}+\frac{1}{2(\beta-\alpha) \operatorname{det} B}\left[\alpha \beta\left|B^{(1)}\right|^{2}-\left|B^{(2)}\right|^{2}-\sqrt{g_{\alpha}(B)}\right], \\
& r_{2}^{\alpha}(B)=\frac{1}{2}+\frac{1}{2(\beta-\alpha) \operatorname{det} B}\left[\alpha \beta\left|B^{(1)}\right|^{2}-\left|B^{(2)}\right|^{2}+\sqrt{g_{\alpha}(B)}\right] .
\end{aligned}
$$

Finally $\Gamma_{\alpha}$ is the set of matrices

$$
\Gamma_{\alpha}=\left\{B \in \mathbf{R}^{2 \times 2}: g_{\alpha}(B) \geq 0, h_{\alpha}(B) \leq 0\right\} .
$$

Theorem 1. Assume that the function $\varphi(x, u, \cdot)$ is convex for a.e. $x \in \Omega$ and all $u$. Then the semiconvex envelope $W_{\alpha}^{\sharp}$ is given by

$$
\begin{aligned}
& W_{\alpha}^{\sharp}(x, U, A, t)= \\
& \begin{cases}\varphi\left(x, U^{(1)}, A^{(1)}\right), & \text { if } A \in \Lambda_{\beta}, t=0 \\
0, & \text { if } A \in \Lambda_{\alpha}, t=1 \\
(1-t) \varphi\left(x, U^{(1)},-\frac{1}{(1-t)(\beta-\alpha)}\left(\alpha A^{(1)}+T A^{(2)}\right)\right), & \text { if } A \in \Gamma_{\alpha} \backslash\left(\Lambda_{\alpha} \cup \Lambda_{\beta}\right), t \in\left[r_{1}^{\alpha}(A), r_{2}^{\alpha}(A)\right] \\
+\infty, & \text { otherwise. }\end{cases}
\end{aligned}
$$

Moreover, in computing $W_{\alpha}^{\sharp}$ the optimal microstructures are first order-laminates if $t=r_{1}^{\alpha}(A), r_{2}^{\alpha}(A)$ and second-order laminates if $t \in\left(r_{1}^{\alpha}(A), r_{2}^{\alpha}(A)\right)$.

The proof we give here for this theorem partially follows the ideas of $[8,23]$. The explicit expression of the optimal laminates is also known and it is included in the proof. We would like to emphasize that the computation of the optimal microstructures is new and particular of the situation that we consider in this paper.

Proof. We can assume without loss of generality that $W_{\alpha}$ does not depend on $(x, u)$, i.e. $W_{\alpha}=W_{\alpha}(\nabla u)$ (this happens when $\varphi=\varphi(\nabla u))$. The case $A \in \Lambda_{\alpha} \cup \Lambda_{\beta}$ is trivial so that we assume $A \in \Gamma_{\alpha} \backslash\left(\Lambda_{\alpha} \cup \Lambda_{\beta}\right)$.

It is clear that the set where $W_{\alpha}^{\sharp}$ ( is finite is $\Lambda_{\alpha} \cup \Lambda_{\beta}$. Thus, to compute the infimum (3) we just need to consider measures with support contained in that set, barycenter $A$ and with volume fraction $t$. We divide the proof into three steps.

Step 1 (previous considerations). Given a Young measure $\nu$ with support contained in the set $\Lambda_{\alpha} \cup \Lambda_{\beta}$ and such that

we can decompose

$$
t=\int_{\mathbf{M}^{2 \times 2}} V_{\alpha}(F) \mathrm{d} \nu(F)
$$

$$
\nu=t \nu_{\alpha}+(1-t) \nu_{\beta}
$$

where $\nu_{\alpha}$ and $\nu_{\beta}$ are probabilities measures with support contained in $\Lambda_{\alpha}, \Lambda_{\beta}$ respectively. We call

$$
A_{\alpha}=\int_{\Lambda_{\alpha}} F \mathrm{~d} \nu_{\alpha}(F) \in \Lambda_{\alpha}, \quad A_{\beta}=\int_{\Lambda_{\beta}} F \mathrm{~d} \nu_{\beta}(F) \in \Lambda_{\beta} .
$$


Due to the weak continuity of the determinant we have that

$$
\operatorname{det}\left(\int_{\mathbf{M}^{2 \times 2}} F \mathrm{~d} \nu(F)\right)=\int_{\mathbf{M}^{2 \times 2}} \operatorname{det}(F) \mathrm{d} \nu(F) .
$$

Indeed, this equality is not true in general, but it is in this case due to the existence of a generating sequence for the Young measure with equi-integrable square of the gradient. Exploiting this inequality and typical algebraic formulas for the determinant of matrices in $\mathbf{M}^{2 \times 2}$ it is not hard to check the inequality

$$
\operatorname{det}\left(A_{\alpha}-A_{\beta}\right) \leq 0
$$

Using the decomposition it is an easy calculation to obtain that

$$
A_{\alpha}=\left(\begin{array}{c}
z \\
\alpha T z
\end{array}\right), \quad A_{\beta}=\left(\begin{array}{c}
w \\
\beta T w
\end{array}\right)
$$

where

$$
\begin{aligned}
z & =\frac{1}{t(\beta-\alpha)}\left(\beta A^{(1)}+T A^{(2)}\right), \\
w & =-\frac{1}{(1-t)(\beta-\alpha)}\left(\alpha A^{(1)}+T A^{(2)}\right) .
\end{aligned}
$$

Notice that matrices $A_{\alpha}$ and $A_{\beta}$ are uniquely determined by $A$ and $t$ and are independent of $\nu$ itself.

Again by an elementary computation we obtain that

$$
\operatorname{det}\left(A_{\alpha}-A_{\beta}\right)=\left|A_{1}\right|^{2} \frac{\alpha}{t^{2}}+\left|A_{2}\right|^{2} \frac{\beta}{(1-t)^{2}}-A_{1} \cdot A_{2} \frac{\alpha+\beta}{t(1-t)},
$$

where

$$
A_{1}=\frac{1}{\beta-\alpha}\left(\beta A^{(1)}+T A^{(2)}\right), \quad A_{2}=-\frac{1}{\beta-\alpha}\left(\alpha A^{(1)}+T A^{(2)}\right) .
$$

Following [8] and exploiting the inequality (4)

$$
\left|A_{1}\right|^{2} \frac{\alpha}{t^{2}}+\left|A_{2}\right|^{2} \frac{\beta}{(1-t)^{2}}-A_{1} \cdot A_{2} \frac{\alpha+\beta}{t(1-t)} \leq 0
$$

we obtain that in order to exist any $t \in(0,1)$ verifying such an inequality the two conditions

$$
g_{\alpha}(A) \geq 0, \quad h_{\alpha}(A) \leq 0
$$

must hold, that is to say

$$
A \in \Gamma_{\alpha}
$$

Step 2 (a lower bound for the semiconvex envelope $W_{\alpha}^{\sharp}$ ). Using the decomposition of the Young measure $\nu$, the cost functional can be rewritten as

$$
t \int_{\Lambda_{\alpha}} W_{\alpha}(F) \mathrm{d} \nu_{\alpha}(F)+(1-t) \int_{\Lambda_{\beta}} W_{\alpha}(F) \mathrm{d} \nu_{\beta}(F)=(1-t) \int_{\Lambda_{\beta}} \varphi\left(F^{(1)}\right) \mathrm{d} \nu_{\beta}(F)
$$

and having in mind that $\varphi$ is convex we can use the Jensen's inequality to obtain that this expression is greater or equal than

$$
m_{A}(t)=(1-t) \varphi\left(A_{\beta}^{(1)}\right)=(1-t) \varphi\left(-\frac{1}{(1-t)(\beta-\alpha)}\left(\alpha A^{(1)}+T A^{(2)}\right)\right) .
$$


Then the value $m_{A}(t)$ provides a lower bound for the constrained semiconvex envelope $W_{\alpha}^{\sharp}(A, t)$. Of course if $(A, t)$ is such that $(5)$ is not verified then

$$
m_{A}(t)=+\infty
$$

and consequently

$$
W_{\alpha}^{\sharp}(A, t)=+\infty .
$$

In this case, given $A \in \Gamma_{\alpha}$, it is an elementary computation to check that those $t^{\prime} s$ verifying (5) are the values in the interval

$$
t \in\left[r_{1}(A), r_{2}(A)\right] .
$$

Step 3 (attaining of the lower bound). If we prove that there exists an admissible homogenous Young measure, $\nu$, with barycenter $A$ and such that

$$
m_{A}(t)=\int_{\mathbf{M}^{2 \times 2}} W_{\alpha}(F) \mathrm{d} \nu(F),
$$

and

$$
t=\int_{\mathbf{M}^{2 \times 2}} V_{\alpha}(F) \mathrm{d} \nu(F),
$$

for any $A \in \Gamma_{\alpha}$ and $t \in\left[r_{1}(A), r_{2}(A)\right]$, then we have proved the result. We will show that such a measure exists in the form of a finite order-laminate, more concretely, a first-order laminate if $t=r_{i}(A), i=1,2$, or a second-order laminate if $t \in\left(r_{1}(A), r_{2}(A)\right)$.

For the case in which $t$ coincides with any of the extreme points of the interval $\left[r_{1}(A), r_{2}(A)\right]$, we obtain equality in (5) and also in (4) so that it is clear that

$$
\nu=t \delta_{A_{\alpha}}+(1-t) \delta_{A_{\beta}}
$$

is a first-order laminate and it attains the bound. In other case, we find the optimal microstructure in the form of the second-order laminate

$$
\nu=s \delta_{C}+(1-s)\left(\sigma \delta_{B}+(1-\sigma) \delta_{A_{\beta}}\right),
$$

with $(1-s)(1-\sigma)=(1-t)$ and

$$
\begin{gathered}
A_{\beta}=\left(\begin{array}{c}
w \\
\beta T w
\end{array}\right), \quad w=\frac{1}{1-t} A_{2}, \\
B=\left(\begin{array}{c}
z_{1} \\
\alpha T z_{1}
\end{array}\right), \quad z_{1}=\frac{1-r_{2}^{\alpha}(A)}{(1-t) r_{2}^{\alpha}(A)} A_{1}, \\
C=\left(\begin{array}{c}
z_{2} \\
\alpha T z_{2}
\end{array}\right), \quad z_{2}=\frac{1}{s} \frac{r_{2}^{\alpha}(A)-t}{t(1-t) r_{2}^{\alpha}(A)} A_{1} .
\end{gathered}
$$

The following facts are elementary (but tedious)to check:

1. $A=\int_{\mathbf{M}^{2 \times 2}} F \mathrm{~d} \nu(F)$;

2. $m_{t}(A)=\int_{\mathbf{M}^{2 \times 2}} W(F) \mathrm{d} \nu(F)$;

3. $\operatorname{det}\left(A_{\beta}-B\right)=0$;

4. there exists $\sigma \in(0,1)$ such that

$$
\operatorname{det}\left(C-\left(\sigma B+(1-\sigma) A_{\beta}\right)\right)=0 .
$$

For the last item we just impose (6) and we get the polynomial at (5) compose with a function depending on $\sigma$. 
It is important to mention here that we are able to compute the relaxed density $W_{\alpha}^{\sharp}$ for any objective convex function $\varphi$ because we define the cost as zero on the manifold $\Lambda_{\alpha}$. An extension of this computation to the case in which we consider a different general function on that manifold is not clear as it is shown in [23].

As the integrand $W_{\alpha}$ is not a Carathéodory function, the classical relaxation results in the Calculus of Variations do not apply to this situation. Nevertheless we can prove a relaxation result taking advantage of the structure of the set where $W_{\alpha}$ is finite, or essentially of the set $\Lambda_{\alpha} \cup \Lambda_{\beta}$. We introduce the following notation.

$$
\begin{gathered}
m_{\alpha}=\inf \left\{I_{\alpha}(U)=\int_{\Omega} W_{\alpha}(x, U(x), \nabla U(x)) \mathrm{d} x: U \text { admissible, } \int_{\mathbf{M}^{2 \times 2}} V_{\alpha}(\nabla U(x)) \mathrm{d} x=\delta\right\}, \\
m_{\alpha}^{\sharp}=\inf \left\{I_{\alpha}^{\sharp}(U)=\int_{\Omega} W_{\alpha}^{\sharp}(x, U(x), \nabla U(x), t(x)) \mathrm{d} x: U\right. \text { admissible, } \\
\left.t \in L^{\infty}(\Omega), 0 \leq t(x) \leq 1, \int_{\Omega} t(x) \mathrm{d} x=\delta\right\}, \\
\tilde{m}_{\alpha}=\inf \left\{\tilde{I}_{\alpha}(\nu)=\int_{\Omega} \int_{\mathbf{M}^{2 \times 2}} W_{\alpha}(x, U(x), F) \mathrm{d} \nu_{x}(F) \mathrm{d} x: \nu \text { admissible }\right\}
\end{gathered}
$$

where $U$ admissible means, $U \in H^{1}\left(\Omega ; \mathbf{R}^{2}\right)$ with $U^{(1)}=u_{0}$ on $\partial \Omega$, and the admissible $\nu$ 's for this last optimization problem are those Young measure $\nu$ such that

$$
\nabla U(x)=\int_{\mathbf{M}^{2 \times 2}} F \mathrm{~d} \nu_{x}(F)
$$

with $U$ admissible and

$$
\int_{\Omega} \int_{\mathbf{M}^{2 \times 2}} V_{\alpha}(F) \mathrm{d} \nu_{x}(F) \mathrm{d} x=\delta .
$$

Theorem 2. The equalities

$$
m_{\alpha}=m_{\alpha}^{\sharp}=\tilde{m}_{\alpha}
$$

hold. Moreover the infima $m_{\alpha}^{\sharp}, \tilde{m}_{\alpha}$ are attained.

Recall that in our case are rather obvious the inequalities

$$
\tilde{m}_{\alpha} \leq m_{\alpha}^{\sharp} \leq m_{\alpha}
$$

(see [21]). The key point of the relaxation result is to prove that any Young measure with support contained in the set $\Lambda_{\alpha} \cup \Lambda_{\beta}$ can be generated by a sequence of gradients taking values on that set. Noticing this we make sure that there is no gap between $m_{\alpha}$ and $\tilde{m}_{\alpha}$ and we get the equality of Theorem 2 . The proof is of technical nature, based on the fact that the set $\Lambda_{\alpha} \cup \Lambda_{\beta}$ comes from a linear elliptic PDE, and we do not include it here, the reader is referred to [8] for a proof in the unconstrained case. In the volume constrained case the proof would be identical just modifying appropriately the essential sets $\Omega_{j}$ in order to impose the volume constraint.

The fact that the infima $m_{\alpha}^{\sharp}$ and $\tilde{m}_{\alpha}$ are attained can be obtained using the direct method of the Calculus of Variations and noticing that the coercivity is a consequence of coercivity for problem $\left(P V_{\alpha}\right)$ (see also [8], Lem. 3.2) and the weak lower semicontinuity is a consequence of the jointly convex character of $W_{\alpha}^{\sharp}$, as this density is defined as a semiconvex envelope. See [17] for the definition of jointly convexity in the context of variational problems with constraints like those that we consider in this paper. 


\section{Relaxation of problem $(P V)$}

In this section we would like to carry out an analysis of relaxation for the variational reformulation of problem $(P)$ analogous to the analysis of Section 3 for problem $\left(P_{\alpha}\right)$. In this case we find the additional difficulty of the lack of coercivity of problem $(P)$, which will have consequences in the analysis as we will see below. Let us begin by defining the appropriate semiconvex envelope in this case. Similarly to the case $\alpha>0$, we define

$$
W^{\sharp}(x, U, A, t)=\inf \left\{\int_{\mathbf{M}^{2 \times 2}} W(x, U, F) \mathrm{d} \nu(F): \nu \in \mathcal{A}(A, t)\right\}
$$

where $\mathcal{A}(A, t)$ is the set of homogenous gradient Young measures $\nu$ such that

$$
A=\int_{\mathbf{M}^{2 \times 2}} F \mathrm{~d} \nu(F)
$$

and

Now we call

$$
t=\int_{\mathbf{M}^{2 \times 2}} V(F) \mathrm{d} \nu(F) .
$$

and for a matrix $A \in \Gamma \backslash\left(\Lambda_{0} \cup \Lambda_{\beta}\right)$ we define

$$
\Gamma=\left\{A \in \mathbf{M}^{2 \times 2}:\left|A^{(2)}\right|^{2} \leq \beta \operatorname{det} A\right\}
$$

$$
t_{A}=1-\frac{\left|A^{(2)}\right|^{2}}{\beta \operatorname{det} A}
$$

Notice that if $A \in \Gamma \backslash\left(\Lambda_{0} \cup \Lambda_{\beta}\right)$ then $\operatorname{det} A \neq 0$, and therefore the definition of $t_{A}$ makes sense. The following result establishes the explicit expression of $W^{\sharp}$.

Theorem 3. Assume that for every pair $(x, u)$ the function

$$
\varphi(x, u, \cdot)
$$

is convex. Then

$$
W^{\sharp}(x, U, A, t)= \begin{cases}\varphi\left(x, U^{(1)}, A^{(1)}\right), & \text { if } A \in \Lambda_{\beta}, t=0 \\ 0, & \text { if } A \in \Lambda_{0}, t=1 \\ (1-t) \varphi\left(x, U^{(1)},-\frac{1}{(1-t) \beta} T A^{(2)}\right), & \text { if } A \in \Gamma \backslash\left(\Lambda_{0} \cup \Lambda_{\beta}\right), t \in\left(0, t_{A}\right] \\ +\infty, & \text { otherwise. }\end{cases}
$$

Moreover in computing $W^{\sharp}$ there is an optimal microstructure in the form of a second-order laminate if $t \in$ $\left(0, t_{A}\right)$, and a first-order laminate for $t_{A}$.

Proof. The proof of this theorem follows the line of the one for Theorem 1, but it is slightly different and we think it is worth for the reader including it. We can assume without loss of generality that $W$ does not depend on $(x, U)$, i.e. $\varphi$ does not depend on $(x, u), \varphi=\varphi(\nabla u)$. It is clear by the definition of $W$ that for computing the envelope

$$
W^{\sharp}(A, t)=\min \left\{\int_{\mathbf{M}^{2 \times 2}} W(F) \mathrm{d} \nu(F): \nu \in \mathcal{A}(A, t)\right\}
$$

we just need to consider Young measures supported where $W$ is finite, i.e. the set $\Lambda_{0} \cup \Lambda_{\beta}$. The border-line cases $t=0,1$ are trivial so that we assume $A \notin\left(\Gamma_{0} \cup \Gamma_{\beta}\right)$. 
Step 1 (previous considerations). We can decompose

$$
\nu=t \nu_{0}+(1-t) \nu_{\beta}
$$

where $\nu_{0}, \nu_{\beta}$ are probability measures supported in $\Lambda_{0}, \Lambda_{\beta}$ respectively. We call

$$
\begin{aligned}
& A_{0}=\int_{\Lambda_{0}} F \mathrm{~d} \nu_{0}(F) \in \Lambda_{0}, \\
& A_{\beta}=\int_{\Lambda_{\beta}} F \mathrm{~d} \nu_{\beta}(F) \in \Lambda_{\beta} .
\end{aligned}
$$

As before, the equality

$$
\operatorname{det}\left(\int_{\mathbf{M}^{2 \times 2}} F \mathrm{~d} \nu(F)\right)=\int_{\mathbf{M}^{2 \times 2}} \operatorname{det} F \mathrm{~d} \nu(F),
$$

holds for any admissible measure $\nu$ for the minimization problem (7). Exploiting this identity we obtain the inequality

$$
\operatorname{det}\left(A_{0}-A_{\beta}\right) \leq 0 .
$$

It is an elementary computation to check that

$$
A_{0}=\left(\begin{array}{c}
z \\
0
\end{array}\right)
$$

and

where

$$
A_{\beta}=\left(\begin{array}{c}
w \\
\beta T w
\end{array}\right)
$$

Again by an elementary computation

$$
z=\frac{1}{t}\left(A^{(1)}+\frac{1}{\beta} T A^{(2)}\right), \quad w=-\frac{1}{\beta(1-t)} T A^{(2)} .
$$

$$
\operatorname{det}\left(A_{0}-A_{\beta}\right)=-\frac{1}{t(1-t)}\left(\operatorname{det} A-\frac{1}{(1-t) \beta}\left|A^{(2)}\right|^{2}\right),
$$

so that the condition

$$
\operatorname{det}\left(A_{0}-A_{\beta}\right) \leq 0
$$

can be rewritten as

$$
\frac{1}{(1-t) \beta}\left|A^{(2)}\right|^{2}-\operatorname{det} A \leq 0 .
$$

It is clear that there exists $t \in[0,1]$ satisfying that condition if and only if

$$
0<\frac{\left|A^{(2)}\right|^{2}}{\beta \operatorname{det} A} \leq 1
$$

that is to say, if $A \in \Gamma$. Actually the inequality

$$
0<\frac{\left|A^{(2)}\right|^{2}}{\beta \operatorname{det} A}
$$

is trivial. 
Step 2 (a lower bound for the semiconvex envelope $W^{\sharp}$ ). Using the decomposition of the measure $\nu$, the cost functional can be rewritten as

$$
t \int_{\Lambda_{0}} W(F) \mathrm{d} \nu_{0}(F)+(1-t) \int_{\Lambda_{\beta}} W(F) \mathrm{d} \nu_{\beta}(F)=(1-t) \int_{\Lambda_{\beta}} \varphi\left(F^{(1)}\right) \mathrm{d} \nu_{\beta}(F)
$$

and having in mind that $\varphi$ is convex we can use the Jensen's inequality to obtain that this expression is greater or equal than

$$
(1-t) \varphi\left(A_{\beta}^{(1)}\right)=(1-t) \varphi\left(-\frac{1}{(1-t) \beta} T A^{(2)}\right)
$$

whenever $A \in \Gamma$ and $t \in\left(0,1-\frac{\left|A^{(2)}\right|^{2}}{\beta \operatorname{det} A}\right]$ (this is the set of $t$ 's that can be attained by Young measures with barycenter $A$ by Step 1). Therefore

where

$$
W^{\sharp}(A, t) \geq m_{A}(t),
$$

$$
m_{A}(t)= \begin{cases}(1-t) \varphi\left(-\frac{1}{(1-t) \beta} T A^{(2)}\right), & \text { if } A \in \Gamma, t \in\left(0,1-\frac{\left|A^{(2)}\right|^{2}}{\beta \operatorname{det} A}\right] \\ +\infty, & \text { otherwise. }\end{cases}
$$

Step 3 (attaining of the lower bound). For proving this, it is enough to prove that for any matrix $A \in \Gamma$ and any $t \in\left(0,1-\frac{\left|A^{(2)}\right|^{2}}{\beta \operatorname{det} A}\right]$ there exists a homogeneous Young measure (a microstructure) $\nu$ supported in $\Lambda_{0} \cup \Lambda_{\beta}$ with barycenter $A$ and

such that

$$
\int_{\mathbf{M}^{2 \times 2}} V(F) \mathrm{d} \nu(F)=t
$$

If $t=t_{A}$ then the first order laminate

$$
\int_{\mathbf{M}^{2 \times 2}} W(F) \mathrm{d} \nu(F)=m_{A}(t) .
$$

$$
t_{A} \delta_{A_{0}}+\left(1-t_{A}\right) \delta_{A_{\beta}}
$$

is an optimal microstructure. This is a first-order laminate because when $t=t_{A}$ the inequality (8) becomes a equality. If $t \in\left(t_{A}, 1\right)$, we are going to show that there exists an admissible microstructure verifying (9) in the form of a second-order laminate

$$
\nu=s \delta_{C}+(1-s)\left(\sigma \delta_{B}+(1-\sigma) \delta_{A_{\beta}}\right)
$$

where $A_{\beta} \in \Lambda_{\beta}$ and $B, C \in \Lambda_{0}$ and very important $(1-s)(1-\sigma)=(1-t)$ (this determines $s$ as a function of $\sigma$ bearing in mind that $t$ is fixed).

Let us take

$$
\begin{aligned}
& A_{\beta}=\left(\begin{array}{c}
-\frac{1}{\beta(1-t)} T A^{(2)} \\
\frac{1}{(1-t)} A^{(2)}
\end{array}\right), \\
& B=\left(\begin{array}{c}
\frac{1-t_{A}}{t_{A}(1-t)}\left(A^{(1)}+\frac{1}{\beta} T A^{(2)}\right) \\
0
\end{array}\right) \text {, }
\end{aligned}
$$

and

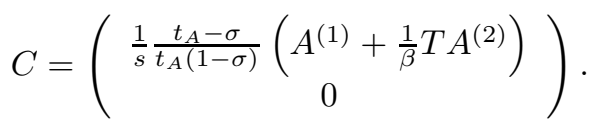

It is not hard to check the following:

$$
\text { 1. } A=\int_{\mathbf{M}^{2 \times 2}} F \mathrm{~d} \nu(F) \text {; }
$$




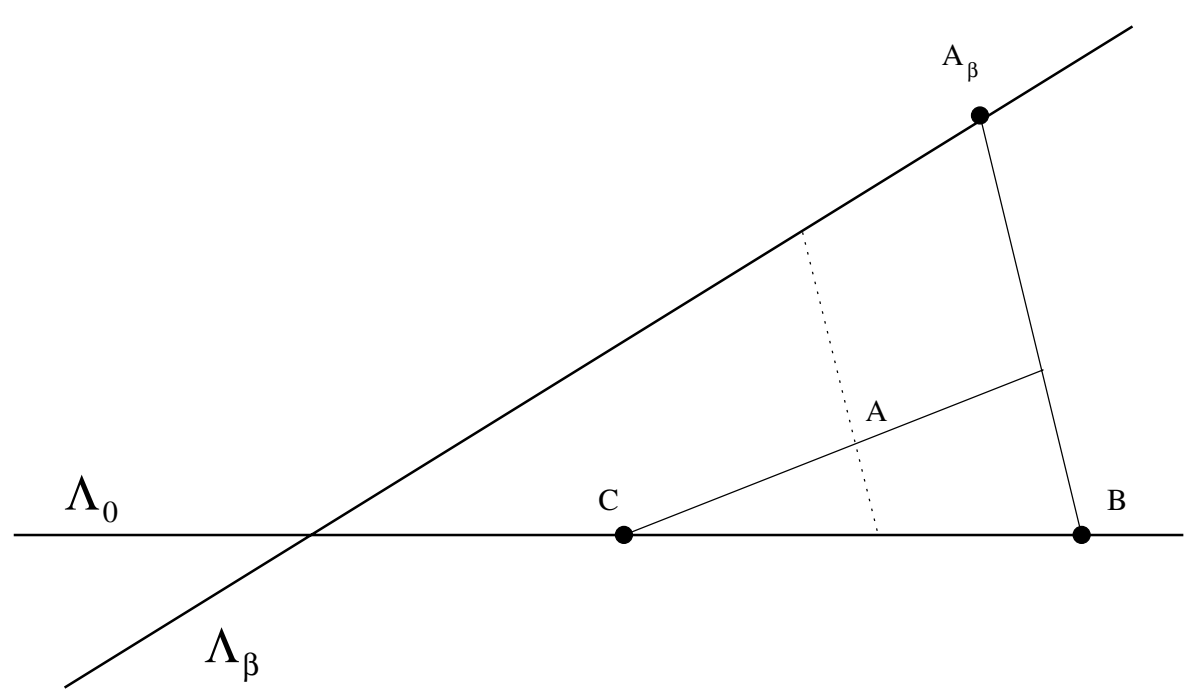

FiguRE 1. Optimal second-order laminate $\nu$.

2. $m_{A}(t)=\int_{\mathbf{M}^{2 \times 2}} W(F) \mathrm{d} \nu(F) ;$
3. $\operatorname{det}\left(A_{\beta}-B\right)=0$.

We have to prove that $\nu$ is a second-order laminate, and this is so if

$$
\operatorname{det}\left(C-\left(\sigma B+(1-\sigma) A_{\beta}\right)=0\right.
$$

It is a tedious but elementary computation to check that this condition is verified taking

$$
\sigma=\frac{1}{2}\left(\frac{\beta \operatorname{det} A}{\left|A^{(2)}\right|^{2}}(1-t)+(2 t-1)\right) .
$$

For finishing having in mind that $A \in \Gamma$ is easy to see that $\sigma \in(0,1)$.

If we call, as in the previous section,

$$
\begin{gathered}
m=\inf \left\{I(U)=\int_{\Omega} W(x, U(x), \nabla U(x)) \mathrm{d} x: U \text { admissible, } \int_{\mathbf{M}^{2 \times 2}} V(\nabla U(x)) \mathrm{d} x=\delta\right\}, \\
m^{\sharp}=\inf \left\{I^{\sharp}(U, t)=\int_{\Omega} W^{\sharp}(x, U(x), \nabla U(x), t(x)) \mathrm{d} x: U\right. \text { admissible, } \\
\left.t \in L^{\infty}(\Omega), 0 \leq t(x) \leq 1, \int_{\Omega} t(x) \mathrm{d} x=\delta\right\}, \\
\tilde{m}=\inf \left\{\tilde{I}(\nu)=\int_{\Omega} \int_{\mathbf{M}^{2 \times 2}} W(x, U(x), F) \mathrm{d} \nu_{x}(F) \mathrm{d} x: \nu \text { admissible }\right\}
\end{gathered}
$$

where $U$ admissible means $U \in H^{1}\left(\Omega ; \mathbf{R}^{2}\right)$ with $U^{(1)}=u_{0}$ on $\partial \Omega$, and the admissible $\nu$ 's for this last optimization problem are those Young measure $\nu$ such that

$$
\nabla U(x)=\int_{\mathbf{M}^{2 \times 2}} F \mathrm{~d} \nu_{x}(F),
$$


with $U$ admissible and

Again the inequalities

$$
\int_{\Omega} \int_{\mathbf{M}^{2 \times 2}} V(F) \mathrm{d} \nu_{x}(F) \mathrm{d} x=\delta .
$$

$$
\tilde{m} \leq m^{\sharp} \leq m
$$

hold. For having a relaxation result we need to have equality and to show that $\tilde{m}$ and $m^{\sharp}$ are attained. We explore this here and we are able prove the equality of the three infima when the function $\varphi$ does not depend on $u$, however we are not able to prove that the infima $\tilde{m}$ and $m^{\sharp}$ are attained. Again the difficulty that we find is the lack of coercivity of the problem: given a minimizing sequence, $\left\{U_{j}\right\}$, for the infimum $m^{\sharp}$ by the the structure of the set $\Gamma$ we can prove that $\left\{\nabla U_{j}^{(2)}\right\}$ is bounded in $L^{2}$ (see Lem. 3.1 in [8] for a proof in the non-degenerate case that could be extended easily to our situation here), so that there exists a subsequence (that we still denote the same) and $v \in H^{1}(\Omega)$ such that (maybe after extracting certain constants)

$$
U_{j}^{(2)} \rightarrow v, \quad \text { weakly in } H^{1} .
$$

The matter is that we do not know what happens with the sequence of first components, $\left\{U_{j}^{(1)}\right\}$, as we do not have any condition implying the boundness of this sequence in any norm. Anyhow, in order to prove existence we do not need that much, it would be enough to conclude the existence of a function $u \in H^{1}(\Omega)$, with $u=u_{0}$ on $\partial \Omega$, such that

$$
(\nabla u(x), \nabla v(x)) \in \Gamma, \quad \text { a.e. } x \in \Omega .
$$

Unfortunately, a statement like this, it is not expectable to be true in general, taking into accounts the results on homogenization of problems in perforated domains where non-local effects appear, see the works $[13,14]$ and the references therein. This really shows the tremendous theoretical difficulty of the problem we are considering.

The following lemma is itself a version of the equality in (12). The proof is technical an particular of the situations that we are considering here.

Lemma 4. Let us assume that $\varphi$ does not depend on u, i.e. $\varphi=\varphi(x, \nabla u)$, and let $\nu=\left\{\nu_{x}\right\}_{x \in \Omega}$ be a $H^{1}$-Young measure verifying

1. $\nu_{x}$ is a finite-order laminate;

2. $\operatorname{supp}\left(\nu_{x}\right) \subset \Lambda_{0} \cup \Lambda_{\beta}$;

3. $\int_{\Omega} \int_{\mathbf{M}^{2 \times 2}} V(F) \mathrm{d} \nu_{x}(F) \mathrm{d} x=\delta$.

Then there exists a sequence $\left\{\bar{U}_{j}\right\}$, such that

1. $\nabla \bar{U}_{j}(x) \in \Lambda_{0} \cup \Lambda_{\beta}$ a.e. $x \in \Omega$;

2. $\lim _{j \rightarrow \infty} \int_{\Omega} W\left(x, \nabla \bar{U}_{j}(x)\right) \mathrm{d} x=\int_{\Omega} \int_{\mathbf{M}^{2 \times 2}} W(x, F) \mathrm{d} \nu_{x}(F) \mathrm{d} x$;

3. $\int_{\Omega} V\left(\nabla \bar{U}_{j}(x)\right) \mathrm{d} x=\delta$ for all $j \geq 1$;

4. finally, if

then

$$
\nabla U(x)=\int_{\mathbf{M}^{2 \times 2}} F \mathrm{~d} \nu_{x}(F),
$$

$$
\bar{U}_{j}^{(2)} \rightarrow U^{(2)}, \quad \text { weakly } H^{1}
$$

Proof. Let $\nu=\left\{\nu_{x}\right\}_{x \in \Omega}$ a $H^{1}$-Young measure in the conditions of the statement, and $U \in H^{1}\left(\Omega ; \mathbf{R}^{2}\right)$ such that

$$
\nabla U(x)=\int_{\mathbf{M}^{2 \times 2}} F \mathrm{~d} \nu_{x}(F) .
$$


There exists a sequence $\left\{U_{j}\right\} \subset H^{1}\left(\Omega ; \mathbf{R}^{2}\right)$ (see [21]) such that $\nu$ is the Young measure associated to $\left\{\nabla U_{j}\right\}$ and

1. $\left\{\left|\nabla U_{j}\right|^{2}\right\}$ is equi-integrable;

2. $U_{j}^{(1)}=u_{0}$ on $\partial \Omega$ for all $j \geq 1$.

If we call

$$
\begin{gathered}
\Omega_{j}=\left\{x \in \Omega: \nabla U_{j}(x) \notin \Lambda_{0} \cup \Lambda_{\beta}\right\}, \\
\omega_{j}=\left\{x \in \Omega: \nabla U_{j}(x) \in \Lambda_{0}\right\},
\end{gathered}
$$

then by the fact that $\nu$ is a family of finite-order laminates with support contained on $\Lambda_{0} \cup \Lambda_{\beta}$ we can assume that

$$
\left|\Omega_{j}\right| \rightarrow 0,
$$

as $j \rightarrow \infty$, and that $\omega_{j}$ is a Lipschitz domain. We can assume without loss of generality that $\partial \omega_{j} \cap \partial \Omega_{j}=\emptyset$. By hypothesis 3 we can claim that

$$
\left|\omega_{j}\right| \rightarrow \delta
$$

as $j \rightarrow \infty$, and as we are working with finite-order laminates we can assume that $\delta<\left|\omega_{j}\right|$ (for this we just increase the volume fraction of the set $\omega_{j}$, i.e. the size of the set itself, so that it is strictly greater than $\delta$ and it converges to $\delta$ as $j \rightarrow \infty$ ). Now we can modify slightly the sets $\omega_{j}$ to obtain new Lipschitz sets $\bar{\omega}_{j}$ such that

$$
\left|\bar{\omega}_{j}\right|=\delta,
$$

and if we call

then

$$
A_{j}=\left(\omega_{j} \backslash \bar{\omega}_{j}\right) \cup\left(\bar{\omega}_{j} \backslash \omega_{j}\right)
$$

$$
\left|A_{j}\right| \rightarrow 0 \text {. }
$$

We can suppose without loss of generality that the sequence $\left\{\nabla U_{j}\right\}$ is such that we can assume that

$$
\bar{\omega}_{j} \subset \omega_{j}, \quad \bar{\omega}_{j} \cap \Omega_{j}=\emptyset,
$$

for all $j \geq 1$.

Since the set $\bar{\omega}_{j}$ is Lipschitz, we can solve the problem

$$
\begin{cases}-\operatorname{div}\left(\beta \nabla \bar{u}_{j}\right)=0, & \text { in } \Omega \backslash \bar{\omega}_{j}, \\ \bar{u}_{j}=u_{0}, & \text { on } \partial \Omega, \\ \beta \nabla \bar{u}_{j} \cdot n=0, & \text { on } \partial \bar{\omega}_{j} .\end{cases}
$$

Let $\bar{U}_{j}^{(1)} \in H^{1}(\Omega)$ be a function such that

$$
\bar{U}_{j}^{(1)}(x)=\bar{u}_{j}(x)
$$

a.e. $x \in \Omega$ (we can assert that, because the domain $\bar{\omega}_{j}$ is Lipschitz, there exists a $H^{1}$-extension operator from $\bar{\omega}_{j}$ to $\Omega$ ). By the transition boundary condition assumed on the boundary of $\bar{\omega}_{j}$ we have that the function

$$
\chi_{\Omega \backslash \bar{\omega}_{j}} \beta \nabla \bar{U}_{j}^{(1)}
$$

is divergence-free, so that there exists $\bar{U}_{j}^{(2)}$ such that

$$
\bar{E}_{j}(x) \nabla \bar{U}_{j}^{(1)}(x)+T \nabla \bar{U}_{j}^{(2)}(x)=0,
$$

a.e. $x \in \Omega$, where $\bar{E}_{j}(x)=\chi_{\Omega \backslash \bar{\omega}_{j}} \beta$. 
By construction,

and

$$
\nabla \bar{U}_{j}(x) \in \Lambda_{0} \cup \Lambda_{\beta},
$$

$$
\int_{\Omega} V\left(\nabla \bar{U}_{j}(x)\right) \mathrm{d} x=\delta
$$

a.e. $x \in \Omega$ and for all $j \geq 1$.

On the other hand, it is clear that the sequence $\left\{\nabla U_{j}\right\}$ verifies

$$
E_{j}(x) \nabla U_{j}^{(1)}(x)+T \nabla U_{j}^{(2)}(x)=0,
$$

a.e. $x \in \Omega \backslash \Omega_{j}$, where $E_{j}(x)=\chi_{\Omega \backslash \omega_{j}} \beta$.

Now using equations (13) and (14) and that $T$ sends curl-free vector fields to divergence-free vector fields, we get the following

$$
\begin{aligned}
\int_{\Omega}\left(\bar{E}_{j}\left(\nabla \bar{U}_{j}^{(1)}-\nabla U_{j}^{(1)}\right)\right) \cdot\left(\nabla \bar{U}_{j}^{(1)}-\nabla U_{j}^{(1)}\right) \mathrm{d} x & =\int_{\Omega}\left(-\bar{E}_{j}\right) \nabla U_{j}^{(1)} \cdot\left(\nabla \bar{U}_{j}^{(1)}-\nabla U_{j}^{(1)}\right) \mathrm{d} x \\
& =\int_{\Omega}\left(-\bar{E}_{j} \nabla U_{j}^{(1)}-T \nabla U_{j}^{(2)}\right) \cdot\left(\nabla \bar{U}_{j}^{(1)}-\nabla U_{j}^{(1)}\right) \mathrm{d} x .
\end{aligned}
$$

It is easy to check that

$$
\bar{E}_{j}(x) \nabla U_{j}^{(1)}(x)+T \nabla U_{j}^{(2)}(x)=0
$$

a.e. $x \in\left(\Omega \backslash A_{j}\right) \cap\left(\Omega \backslash \Omega_{j}\right)$ and as $A_{j} \cup \Omega_{j} \subset \Omega \backslash \bar{\omega}_{j}$, applying Hölder's inequality we obtain that last integral is

$$
\leq\left(\int_{A_{j} \cap \Omega_{j}}\left|\bar{E}_{j}(x) \nabla U_{j}^{(1)}(x)+T \nabla U_{j}^{(2)}(x)\right|^{2} \mathrm{~d} x\right)^{\frac{1}{2}}\left(\int_{\Omega \backslash \bar{\omega}_{j}}\left|\nabla U_{j}^{(1)}(x)-\nabla \bar{U}_{j}^{(1)}(x)\right|^{2} \mathrm{~d} x\right)^{\frac{1}{2}} .
$$

By the equi-integrability of the sequence $\left\{\left|\nabla U_{j}\right|^{2}\right\}$, and the facts $\left|\Omega_{j}\right| \rightarrow 0$ and $\left|A_{j}\right| \rightarrow 0$ we obtain

$$
\int_{\Omega \backslash \bar{\omega}_{j}}\left|\nabla \bar{U}_{j}^{(1)}-\nabla U_{j}^{(1)}\right|^{2} \mathrm{~d} x \rightarrow 0, \quad \text { as } j \rightarrow \infty .
$$

Now we prove that

$$
\begin{gathered}
\nabla \bar{U}_{j}^{(2)}-\nabla U_{j}^{(2)} \rightarrow 0, \quad \text { strong in } L^{2} . \\
\int_{\Omega}\left|\nabla \bar{U}_{j}^{(2)}-\nabla U_{j}^{(2)}\right|^{2} \mathrm{~d} x=\int_{\Omega}\left(T \nabla \bar{U}_{j}^{(2)}-T \nabla U_{j}^{(2)}, T \nabla \bar{U}_{j}^{(2)}-T \nabla U_{j}^{(2)}\right) \mathrm{d} x
\end{gathered}
$$

adding and subtracting $\bar{E}_{j} \nabla U_{j}^{(1)}$ and $\bar{E}_{j} \nabla \bar{U}_{j}^{(1)}$ is easy to check that the above integral is equal to the sum

$$
\int-\left(\bar{E}_{j} \nabla U_{j}^{(1)}+T \nabla U_{j}^{(2)}, T \nabla \bar{U}_{j}^{(2)}-T \nabla U_{j}^{(2)}\right) \mathrm{d} x+\int_{\Omega}\left(\bar{E}_{j}\left(\nabla U_{j}^{(1)}-\nabla \bar{U}_{j}^{(1)}\right), T \nabla \bar{U}_{j}^{(2)}-T \nabla U_{j}^{(2)}\right) \mathrm{d} x .
$$

Applying Hölder's inequality we obtain

$$
\left\|\nabla \bar{U}_{j}^{(2)}-\nabla U_{j}^{(2)}\right\|_{2} \leq\left(\int_{A_{j} \cup \Omega_{j}}\left|\bar{E}_{j} \nabla U_{j}^{(1)}+T \nabla U_{j}^{(2)}\right|^{2} \mathrm{~d} x\right)^{\frac{1}{2}}+\beta\left(\int_{\Omega \backslash \bar{\omega}_{j}}\left|\nabla \bar{U}_{j}^{(1)}-\nabla U_{j}^{(1)}\right|^{2} \mathrm{~d} x\right)^{\frac{1}{2}} .
$$

The second term converges to zero by (15), and for the first term we have already proved above its convergence to zero. 
By (16) is clear that, maybe after extracting suitable constants,

$$
\bar{U}_{j}^{(2)} \rightarrow U^{(2)}, \quad \text { weakly } H^{1} .
$$

For a.e. $x \in \Omega$, the second projection of the measure $\nu_{x}, \pi_{2} \nu_{x}$ is the probability measure given by

$$
\int_{\mathbf{R}^{2}} \Psi(\lambda) \mathrm{d} \pi_{2} \nu_{x}(F)=\int_{\mathbf{M}^{2 \times 2}} \Psi\left(F^{(2)}\right) \mathrm{d} \nu_{x}(F),
$$

for any $\Psi \in \mathcal{C}_{0}\left(\mathbf{R}^{2}\right)$. Obviously the family $\pi_{2} \nu=\left\{\pi_{2} \nu_{x}\right\}$ is a $H^{1}$-Young measure generated by the sequence of gradients $\left\{\nabla U_{j}^{(2)}\right\}$, so that by (16), the sequence $\left\{\nabla \bar{U}_{j}^{(2)}\right\}$ is also a generating sequence for this Young measure.

If we define the function $\tilde{W}: \Omega \times \mathbf{R}^{2} \rightarrow \mathbf{R} \cup\{+\infty\}$ as

$$
\tilde{W}(x, \lambda)= \begin{cases}\varphi\left(x, \frac{1}{\beta} T \lambda\right), & \text { if } \lambda \neq 0 \\ 0, & \text { if } \lambda=0\end{cases}
$$

we obtain the following

$$
\begin{aligned}
\int_{\Omega} \int_{\mathbf{M}^{2 \times 2}} W(x, F) \mathrm{d} \nu_{x}(F) \mathrm{d} x & =\int_{\Omega} \int_{\mathbf{R}^{2}} \tilde{W}(x, \lambda) \mathrm{d} \pi_{2} \nu_{x}(\lambda) \mathrm{d} x \\
& =\lim _{j \rightarrow \infty} \int_{\Omega} \tilde{W}\left(x, \nabla \bar{U}_{j}^{(2)}(x)\right) \mathrm{d} x \\
& =\int_{\Omega} W\left(x, \nabla \bar{U}_{j}(x)\right) \mathrm{d} x .
\end{aligned}
$$

We have not been able to include the general case in Lemma 4. Our approach to prove this result would work if we have that

$$
\lim _{j \rightarrow \infty}\left\|\bar{U}_{j}^{(1)}-U^{(1)}\right\|_{L^{2}\left(\Omega \backslash \bar{\omega}_{j}\right)}=0
$$

This is true in certain periodic cases (see [5]), but unfortunately it is not true in general (see again the examples in $[13,14])$.

Theorem 5. If $\varphi=\varphi(x, \nabla u)$, then

$$
\tilde{m}=m^{\sharp}=m
$$

hold.

The proof of this theorem is a direct consequence of the inequality (12), and the fact that given an admissible gradient Young measure $\mu=\left\{\mu_{x}\right\}$, there exists another gradient Young measure $\nu=\left\{\nu_{x}\right\}$ such that, each $\nu_{x}$ is a laminate of order less or equal that two and

$$
\int_{\Omega} \int_{\mathbf{M}^{2 \times 2}} \varphi(x, F) \mathrm{d} \nu_{x}(F) \mathrm{d} x \leq \int_{\Omega} \int_{\mathbf{M}^{2 \times 2}} \varphi(x, F) \mathrm{d} \mu_{x}(F) \mathrm{d} x .
$$

The proof of this fact is a part of Step 1 in the proof of Proposition 7.

We have explored here the possibility of proving a relaxation result in terms of gradient Young measures, the idea of this is to enlarge the set of admissible designs to the set of all the gradients Young measures associated to bounded sequences of admissible gradients in our original optimal shape design problem. We have proved a partial result in this direction, and as we have mentioned above Lemma 4, a full result is not clear at all. On the one hand, it is possible to find, based on the examples in $[13,14]$, that there exists sequences of admissible gradients for our original problem (not bounded in the first component) that do not generate any gradient Young measure, so that we maybe have to look for minimizers in bigger sets than that of the admissible gradient Young 
measures. In order to do this we would have to advance in the understanding of Homogenization of problems in perforated domains in the case of Neumann boundary conditions. On the other hand, very often in optimal design problems happens that for establishing a relaxation result we have to use a bigger set than the set where all the potential minimizers really live. The fact that given an admissible gradient Young measure we can approximate it in energy by a sequence of admissible gradients (Lem. 4) may indicate that this is the case here, i.e. that $\tilde{m}$ and $m^{\sharp}$ are actually attained. Although the author suspects that this may depend on the particular election of the objective function $\varphi$.

We would like to finish this section mentioning that an interesting direction of research is to do numerical simulations on this for different objective functions in order to test numerically whether the relaxation that we propose really admits minimizers. The author plans to explore this in the near future.

\section{Approximation}

Along this section we will assume that $\varphi$ does not depend on $u$. As we have mentioned in the Introduction an interesting open problem is whether "the optimal shape design problem $(P V)$ may be approximated by the sequence of problems $\left(P V_{\alpha}\right)$ ". Of course the concept of approximation we would like to mean have to be explicitly clarified, and in particular, as those problems lack of optimal solutions, it seems more convenient to deal with their relaxed formulations. A suitable framework to analyze this question would be that of $\Gamma$-convergence (two basic references on $\Gamma$-convergence are $[12,16])$, and then the question we want to understand would be: does the sequence of relaxed formulations of problems $\left(P V_{\alpha}\right) \Gamma$-converge to the "relaxed" formulation of $(P V)$ ? In this section we focus on this problem. Unfortunately we are not able to provide a positive answer to this, but we provide partial answers that, we think, are interesting to know and of physical interest in themselves. It is important to remark that even if the $\Gamma$-convergence result holds, this is limited by the fact that we are not dealing with an equi-coercive sequence, so that many of the consequences of $\Gamma$-convergence will not hold. We would also like to mention that an interesting simulation problem would be to test the $\Gamma$-convergence result numerically, we plan to do this in the near future as well.

We begin recalling the definition of $\Gamma$-convergence. Given a metric space $X$, we say that a sequence $f_{j}: X \rightarrow$ $\overline{\mathbf{R}} \Gamma$-converges in $X$ to $f_{\infty}: X \rightarrow \overline{\mathbf{R}}$ if for all $x \in X$ we have

1. (liminf inequality) for every sequence $\left\{x_{j}\right\}$ converging to $x$

$$
f_{\infty}(x) \leq \liminf _{j} f_{j}\left(x_{j}\right)
$$

2. (limsup inequality) there exists a sequence $\left\{x_{j}\right\}$ converging to $x$ such that

$$
f_{\infty}(x) \geq \limsup _{j} f_{j}\left(x_{j}\right) .
$$

The function $f_{\infty}$ is called the $\Gamma$-limit of $\left\{f_{j}\right\}$. Here our space is $X=W^{1, p}\left(\Omega ; \mathbf{R}^{2}\right) \times L^{\infty}(\Omega)($ with $p=2)$ equipped with the weak and weak- $\star$ topologies respectively. We want to check whether this definition is verified in our situation. The first important fact that we notice is that pointwise convergence of the integrands $W_{\alpha}^{\sharp}$ to $W^{\sharp}$ holds. Effectively, it is not hard to check that, for a fixed matrix $A$, we have that

$$
r_{1}^{\alpha}(A) \rightarrow 0, \quad r_{2}^{\alpha}(A) \rightarrow t_{A}
$$

as $\alpha \rightarrow 0$. It is also easy to see that

$$
\Gamma_{\alpha} \rightarrow \Gamma
$$

as $\alpha \rightarrow 0$, in the sense that the inequalities defining those sets converge pointwise. Finally, by the continuity assumed on the function $\varphi$, it is clear that

$$
W_{\alpha}^{\sharp}(x, A, t) \rightarrow W^{\sharp}(x, A, t)
$$


as $\alpha \rightarrow 0$, for any matrix $A$ and any $t \in[0,1]$. Further, in the computation of $W_{\alpha}^{\sharp}(x, A, t), W^{\sharp}(x, A, t)$ there is convergence of the optimal microstructures that we use in the proofs of Theorems 1 and 3.

There are situations in $\Gamma$-convergence in which we can obtain a convergence result for integral functionals from the pointwise convergence of the integrands. Unfortunately, this is not the situation here, and the author has not even been able to prove the liminf inequality from this fact. However, it is possible to show a, in our context satisfactory, version of the limsup inequality. This is stated in the next proposition.

We would like to emphasize that we consider the $\Gamma$-convergence problem in $H^{1}\left(\Omega ; \mathbf{R}^{2}\right)$ rather than $L^{2}(\Omega)$. This is due to the fact that we are interested in the optimal designs for $\left(P_{\alpha}\right)$ and the optimal shapes for $(P)$ (or their relaxed formulations). These problems are reformulated as vector variational problems, and in these formulations all the information on the admissible designs and shapes is enclosed in the gradients of the admissible functions for those problems, in the sense that if we have an optimal design or shape we can reproduce the optimal microstructure for the original problem. Hence the relevant objects for our vector variational problems are the gradients of the admissible functions and this is the reason because we find reasonable to formulate the $\Gamma$-convergence problem in a Sobolev space.

Proposition 6. Given $U \in H^{1}\left(\Omega ; \mathbf{R}^{2}\right)$ and $t \in L^{\infty}(\Omega)$ such that

$$
\nabla U(x) \in \Gamma, \quad t(x) \in\left(0, t_{\nabla U(x)}\right]
$$

a.e. $x \in \Omega$, there exists a sequence $\left\{U_{\alpha}\right\}$ in $H^{1}\left(\Omega ; \mathbf{R}^{2}\right)$ such that

$$
\nabla U_{\alpha}(x) \in \Lambda_{\alpha} \cup \Lambda_{\beta},
$$

a.e. $x \in \Omega$ and for all $\alpha \geq 0$, such that

$$
\begin{gathered}
U_{\alpha}^{(2)} \rightarrow U^{(2)}, \quad \text { weakly } H^{1}, \\
t_{\alpha} \rightarrow t, \quad \text { weakly }-\star L^{\infty},
\end{gathered}
$$

where

$$
\begin{gathered}
t_{\alpha}(x)=\chi_{\Omega \backslash \omega_{\alpha}}, \\
\omega_{\alpha}=\left\{x \in \Omega: \alpha \nabla U_{\alpha}^{(1)}+T \nabla U_{\alpha}^{(2)}=0\right\},
\end{gathered}
$$

and moreover

$$
\int_{\Omega} W^{\sharp}(\nabla U(x), t(x)) \mathrm{d} x=\lim _{\alpha \rightarrow 0} \int_{\Omega} W_{\alpha}^{\sharp}\left(\nabla U_{\alpha}(x), t_{\alpha}(x)\right) \mathrm{d} x .
$$

Proof.

Step 1. We prove that there exists a sequence $\left\{V_{j}\right\}$ such that

- $\nabla V_{j}(x) \in \Lambda_{0} \cup \Lambda_{\beta}$, a.e. $x \in \Omega$;

- $\int_{\Omega} V\left(\nabla V_{j}(x)\right) \mathrm{d} x=\delta$ for all $j \geq 1$;

- $\nabla V_{j}^{(2)} \rightarrow \nabla U^{(2)}$ weakly $L^{2}$

- $t_{j}(x)=V\left(\nabla V_{j}(x)\right)=\chi_{\Omega \backslash \omega_{j}} \rightarrow t(x)$ weak- $\star$ in $L^{\infty}(\Omega)$, where

$$
\omega_{j}=\left\{x \in \Omega: \nabla V_{j}(x)=0\right\} ;
$$

5. $\lim _{j \rightarrow \infty} \int_{\Omega} W^{\sharp}\left(\nabla V_{j}(x), t_{j}(x)\right) \mathrm{d} x=\int_{\Omega} W^{\sharp}(\nabla U(x), t(x)) \mathrm{d} x$. 
Furthermore we can assume that $\partial \Omega \cap \partial \omega_{j}=\emptyset$. For proving this, we proceed in the following way. For a.e. $x \in \Omega$ let $\nu_{x}$ be the optimal homogeneous Young measure given by Theorem 3 such that

and

$$
\begin{gathered}
W^{\sharp}(\nabla U(x), t(x))=\int_{\mathbf{M}^{2 \times 2}} W(F) \mathrm{d} \nu_{x}(F), \\
\nabla U(x)=\int_{\mathbf{M}^{2 \times 2}} F \mathrm{~d} \nu_{x}(F),
\end{gathered}
$$

$$
t(x)=\int_{\mathbf{M}^{2 \times 2}} V(F) \mathrm{d} \nu_{x}(F) .
$$

Recall that $\nu_{x}$ is either a dirac mass, a first-order laminate or a second-order laminate. We claim that the family $\nu=\left\{\nu_{x}\right\}$ is a $H^{1}$-Young measure. This is true because the characterization theorem of gradient Young measures [21] is satisfied, namely:

1. the first moment of the family is a gradient by definition;

2. each $\nu_{x}$ is a homogenous Young measure itself;

3. $\int_{\Omega} \int_{\mathbf{M}^{2 \times 2}}|F|^{2} \mathrm{~d} \nu_{x}(F)<+\infty$. Proving this is not difficult but a bit tedious, it can be done using the explicit expression for $\nu_{x}$ given at Theorem 3. It is not hard to check that for any of the possibilities we have for this optimal Young measure, the inequality

$$
\int_{\mathbf{M}^{2 \times 2}}|F|^{2} \mathrm{~d} \nu_{x}(F) \leq C|\nabla U(x)|^{2},
$$

where $C$ is positive constant independent of $x$, holds a.e. $x \in \Omega$.

The Young measure $\nu=\left\{\nu_{x}\right\}$ is in the conditions of Proposition 4, therefore the existence of the sequence $\left\{V_{j}\right\}$ is just a consequence of it.

Step 2. We prove that for any $j \geq 1$ there exists a sequence $\left\{U_{j, \alpha}\right\}$ such that:

1. $\nabla U_{\alpha, j}(x) \in \Lambda_{\alpha} \cap \Lambda_{\beta}$, a.e. $x \in \Omega$, and for all $\alpha>0$;

2. $\nabla U_{\alpha, j}^{(2)} \rightarrow \nabla V_{j}^{(2)}$ strong $L^{2}$ as $\alpha \rightarrow 0$;

3. $\left(\alpha\left(1-t_{j}\right)+\beta t_{j}\right) \nabla U_{\alpha, j}^{(1)} \rightarrow \beta t_{j} \nabla V_{j}^{(1)}$ strong $L^{2}$ as $\alpha \rightarrow 0$;

4. finally $\lim _{\alpha \rightarrow 0} \int_{\Omega} W_{\alpha}^{\sharp}\left(\nabla U_{\alpha, j}(x), t_{j}(x)\right) \mathrm{d} x=\int_{\Omega} W^{\sharp}\left(\nabla V_{j}(x), t_{j}(x)\right) \mathrm{d} x$.

Obviously the last item is a straightforward consequence of items 2 and 3. For the proof, let us consider for each $\alpha>0, U_{\alpha, j}^{(1)}$ the solution of the equation

$$
\begin{cases}-\operatorname{div}\left[\left(\left(\alpha\left(1-t_{j}\right)+\beta t_{j}\right) \nabla u\right]=0,\right. & \text { in } \Omega \\ u=u_{0}, & \text { on } \partial \Omega\end{cases}
$$

This function is divergence free so that there exists $U_{\alpha, j}^{(2)} \in H^{1}(\Omega)$ such that

$$
\left(\alpha\left(1-t_{j}\right)+\beta t_{j}\right) \nabla U_{\alpha, j}^{(1)}(x)+T \nabla U_{\alpha, j}^{(2)}(x)=0,
$$

a.e. $x \in \Omega$.

Now we claim that

and

$$
\left(\alpha\left(1-t_{j}\right)+\beta t_{j}\right) \nabla U_{\alpha, j}^{(1)} \rightarrow \beta t_{j} \nabla V_{j}^{(1)}, \quad \text { strong } L^{2},
$$

$$
\nabla U_{\alpha, j}^{(2)} \rightarrow \nabla V_{j}^{(2)}(x), \quad \text { strong } L^{2} .
$$


Let us prove the first convergence. For this we will use the identity

$$
\beta t_{j}(x) \nabla V_{j}^{(1)}+T \nabla V_{j}^{(2)}(x)=0,
$$

a.e. $x \in \Omega$. Notice that it is enough to show that

$$
\int_{\Omega}\left(\left(\alpha\left(1-t_{j}\right)+\beta t_{j}\right)\left(\nabla V_{j}^{(1)}-\nabla U_{\alpha, j}^{(1)}\right), \nabla V_{j}^{(1)}-\nabla U_{\alpha, j}^{(1)}\right) \mathrm{d} x \rightarrow 0
$$

as $\alpha \rightarrow 0$. To prove this is easy as the previous integral is equal, after adding and subtracting $T \nabla V_{j}^{(2)}$ and $T \nabla U_{\alpha, j}^{(2)}$, to

$$
\int_{\Omega}\left(\left(\alpha\left(1-t_{j}\right)+\beta t_{j}\right) \nabla V_{j}^{(1)}-T \nabla V_{j}^{(2)}, \nabla V_{j}^{(1)}-\nabla U_{\alpha, j}^{(1)}\right) \mathrm{d} x
$$

and this integral converges to zero as $\alpha \rightarrow 0$. This is true because, thanks to the fact the function $\alpha\left(1-t_{j}\right)+\beta t_{j}$ takes the value $\beta$ on $\partial \Omega$, we can use a truncation function to prove that

$$
\left\|\nabla U_{\alpha, j}^{(1)}\right\|_{L^{2}\left(\Omega \backslash \omega_{j} ; \mathbf{R}^{2}\right)}+\alpha^{\frac{1}{2}}\left\|\nabla U_{\alpha, j}^{(1)}\right\|_{\left.L^{2} \Omega \backslash \omega_{j} ; \mathbf{R}^{2}\right)},
$$

is uniformly bounded with respect to $\alpha$ (we refer to Lem. 3.1 .5 for a more detailed proof of this in a similar situation).

The second convergence is a direct consequence of the first one.

Step 3. For finishing the proof is enough to use a diagonal sequence procedure to choose a sequence of the form $\left\{\nabla U_{\alpha(j), j}\right\}$ in the conditions of the statement of the theorem.

The results here are obviously far from being an answer to the question formulated at the beginning of this section, and many things still remain to be understood. For instance, because we do not have a liminf inequality type result, we cannot assert that the infimum value of the relaxed formulations for problems $\left(P V_{\alpha}\right)$ converges to the infimum of the relaxed formulation for problem $(P V)$, so that the limit of this sequence might be smaller than the infimum of $(P V)$.

Acknowledgements. This work has been carried out while the author was a Marie-Curie Research Fellow at the Mathematical Institute of the University of Oxford, the author would like to thank the kind hospitality of the Applied Analysis and Material Science Group and in particular of John M. Ball during this period. The author also appreciates very much the hospitality of Grégoire Allaire and the stimulating conversations with him on the subject of this paper during his visit to École Polytechnique. The author also thanks many interesting conversations on the subject of this paper with Daniel Faraco. Financial support from CEC-Marie-Curie Fellowships Program through contract HPMF-CT-2002-02177, research projects MTM2004-07114 of the MEC and PAI05-027 of Castilla-La Mancha (SPAIN).

\section{REFERENCES}

[1] G. Allaire, Shape optimization by the homogenization method. Springer (2002).

[2] G. Allaire, E. Bonnetier, G. Franfort and F. Jouve, Shape optimization by the homogenization method. Numer. Math. 76 (1997) 27-68.

[3] G. Allaire and R.V. Kohn, Optimal bounds on the effective behauvior of a mixture of two well-odered elastic materials. Quat. Appl. Math. 51 (1993) 643-674.

[4] G. Allaire and R.V. Kohn, Optimal design for minimum weight and compliance in plane stress using extremal microstructures. Europ. J. Mech. A/solids 12 (1993) 839-878.

[5] G. Allaire and F. Murat, Homogenization of the Neumann problem with nonisolated holes. Asymptotic Anal. 7 (1993) $81-95$. With an appendix written jointly with A.K. Nandakumar.

[6] J.C. Bellido, Explicit computation of the relaxed density coming from a three-dimensional optimal design prroblem. Nonlinear Analysis TMA 52 (2003) 1709-1726. 
[7] J.C. Bellido and P. Pedregal, Optimal design via variational principles: the one-dimensional case. J. Math. Pures Appl. 80 (2000) 245-261.

[8] J.C. Bellido and P. Pedregal, Explicit quasiconvexification for some cost functionals depending on the derivatives of the state in optimal design. DCDS-A $\mathbf{8}(2002)$ 967-982.

[9] J.C. Bellido and P. Pedregal, Optimal control via variational principles: the three dimensional case. J. Math. Anal. Appl. 287 (2003) $157-176$.

[10] J.C. Bellido and P. Pedregal, Existence in optimal control with state equation in divergence form via variational principles. $J$. Convex Anal. 10 (2003) 365-378.

[11] M.P. Bendsøe and O. Sigmund, Topology optimization, Theory, methods and applications. Springer-Verlag, Berlin (2003).

[12] A. Braides, $\Gamma$-convergence for beginners, Oxford Lecture Series in Mathematics and its Applications. Oxford University Press, Oxford, 22 (2002).

[13] M. Briane, Homogenization in some weakly connected domains. Ricerche Mat. 47 (1998) 51-94.

[14] M. Briane, Homogenization in general periodically perforated domains by a spectral approach. Calc. Var. Partial Differ. Equat. 15 (2002) 1-24.

[15] A. Cherkaev, Variational methods for structural optimization. Springer (2000).

[16] G. Dal Maso, Introduction to $\Gamma$-convergence. Birkhäuser, Boston, 1993.

[17] I. Fonseca, D. Kinderlehrer and P. Pedregal, Energy functionals depending on elastic strain and chemical composition. Cal. Var. 2 (1994) 283-313.

[18] V. Girault and P.A. Raviart, Finite elements methods for Navier-Stokes equations, Theory and Algorithms. Springer-Verlag, Berlin, Heidelberg, New York, Tokyo (1985).

[19] S. Müller and V. Šverák, Convex integration for lipschitz mappings and counterexamples for regularity. Technical Report 26, Max-Planck Institute for Mathematics in the Sciences, Leipzig (1999).

[20] F. Murat, Contre-exemples pour divers problèmes où le contrôle intervient dans les coefficients. Ann. Mat Pura Appl. 112 (1977) 49-68.

[21] P. Pedregal, Parametrized Measures and Variational Principles. Progress in Nonlinear Partial Differential Equations. Birkhäuser (1997).

[22] P. Pedregal, Optimal design and constrained quasiconvexity. SIAM J. Math. Anal. 32 (2000) 854-869.

[23] P. Pedregal, Constrained quasiconvexification of the square of the gradient of the state in optimal design. Quater. Appl. Math. 62 (2004) 459-470.

[24] L. Tartar, Remarks on optimal design problems, in Homogenization and continuum mechanics, G. Buttazzo, G. Bouchitte, and P. Suchet Eds, Singapure World Scientific (1994) 279-296.

[25] L. Tartar, An introduction to homogenization method in optimal design. Lect. Notes Math. Springer (2000).

[26] V. Šverák, Lower semicontinuity of variational integrals and compesated compactness, in Proc. ICM, S.D. Chatterji Ed., Birkhäuser 2 (1994) 1153-1158. 